\title{
Trigeminal Nerve Atrophy due to Vertebrobasilar Dolichoectasia: A Rare Entity
}

\author{
Ahmad Al Ali ${ }^{1}$, Ihsan F Shanti ${ }^{2}$, Zaynab I Shanti ${ }^{3}$, Bilal F Shanti ${ }^{4 *}$ and \\ Hani Al-Ali ${ }^{5}$ \\ ${ }^{1}$ Specialist Neuroradiologist, Jordanian Ministry of Health, Jordan \\ ${ }^{2}$ The American Clinic for Interventional Pain and Spine, Amman, Jordan \\ ${ }^{3}$ Medical Student, Royal College of Surgeons, Dublin, Ireland \\ ${ }^{4}$ Omnia Pain Consultants, Pain Medicine, Phoenix, Arizona, USA \\ ${ }^{5}$ Consultant Musculoskeletal Radiologist, Medical Director of Rayone Medical \\ Imaging Center, Jordan \\ *Corresponding Author: Bilal F Shanti, Omnia Pain Consultants, Pain Medicine, \\ Phoenix, Arizona, USA.
}

Received: August 16, 2020

Published: September 16, 2020

(C) All rights are reserved by Bilal F Shanti., et al.

\begin{abstract}
The term dolichoectasia implies abnormal dilatation and elongation of an artery or vein. In the brain, this condition most commonly affects the vertebrobasilar system. The exact etiologic mechanism of the disease is unknown. However, many theories are suggested such as hypertension, atherosclerosis, and congenital factors (Marfan's disease, Ehlers Danlos disease, autosomal recessive polycystic kidney disease, Fabry disease and sickle cell anemia).

The disease is asymptomatic in most of the cases and thus detected incidentally. However, in minority of patients the disease might be symptomatic and the clinical manifestations include, but not limited to, stroke, brainstem compression, cranial neuropathies (motor and sensory), and hydrocephalus.

Trigeminal atrophy or hypoplasia is a very uncommon condition, usually described in association with Goldenhar-Gorlin syndrome (a complex congenital anomaly) and has not yet been attributed to vertebrobasilar dolichoectasia.

The current case report highlights this rare association of trigeminal nerve atrophy and vertebrobasilar dolichoectasia, leading to hemifacial and corneal anesthesia.
\end{abstract}

Keywords: Magnetic Resonance Imaging; Trigeminal Atrophy; Vertebrobasilar Dolichoectasia

\section{Introduction}

Vertebrobasilar dolichoectasia (VBD) is a rare vascular pathology characterized by increased length and caliber of the vertebral or basilar arteries with tortuosity, diagnosed according to Smoker's criteria. The known incidence is $0.06-5.8 \%[1,2]$.

In patients with VBD, the neural compression has a slow progression, and the brainstem can functionally accommodate to severe degrees of distortion, which may explain why most patients with VBD are asymptomatic. Thus, clinically overt trigeminal nerve atrophy is a rare entity. Clinically, trigeminal nerve atrophy manifests by unilateral hypoesthesia of face, corneal insensitivity and decreased hearing unilaterally (probably related to the innervation of tensor tympani muscle innervated by affected nerve). Radiologically, decreased nerve thickness and altered signal are features suggestive of chronic atrophy. Few cases of trigeminal neuralgia due to 
vertebrobasilar dolichoectasia have been described in literature. In the current case report, dolichoectatic basilar artery associated with trigeminal nerve atrophy was adequately revealed on imaging.

\section{Case Report}

A 70-year-old obese and hypertensive female patient consulted with pain management clinic in Amman Jordan, with symptoms of loss of sensation over the left half of the face, mainly left upper lip which spread subsequently to the rest of the left side of the face, and with burning sensation. No motor deficits were observed.

The patient had also a previous history of right cerebral stroke seven months prior to the current diagnosis resulting in left lower extremity motor deficit.

On examination, the patient's vitals were stable and general examination of the patient was uneventful except for left lower extremity weakness. Neurological examination reveals hypoesthesia of all branches of left trigeminal nerve corresponding to ophthalmic, maxillary and mandibular divisions, and corneal reflex was diminished. Proprioception for temporomandibular joint was present although the mandible deviated slightly to the left. Rest of the neurological examination was normal. Brain CT scan was unremarkable.

Left trigeminal injection was done 3 weeks prior to MRI, through left sphenopalatine ganglion block - greater palatine foramen approach, with a $1 \mathrm{~mL}$ normal saline and $80 \mathrm{mg}$ Methylprednisolone. A week later, during the follow-up, there was mild to moderate improvement of the patient's symptoms and physical examination. Yet the patient opted to receive a second injection.

Two weeks later, the second injection of the left trigeminal ganglion was performed. Same procedure was carried on. MRI was ordered for further evaluation. A week after the second injection, her symptoms improved and the patient was almost pain-free.

MRI revealed an abnormally dilated tortuous basilar artery, measuring about $6.6 \mathrm{~mm}$ in transverse diameter (Figure 1). The artery courses to the left cerebellopontine angle before turning back to the midline anterior to pons, causing compression upon the left anterolateral aspect of the brainstem and the origin of the left trigeminal nerve. The dilated tortuous basilar artery is seen at the level of the floor of the third ventricle (Figure 2). There was no definite evidence of thrombosis.

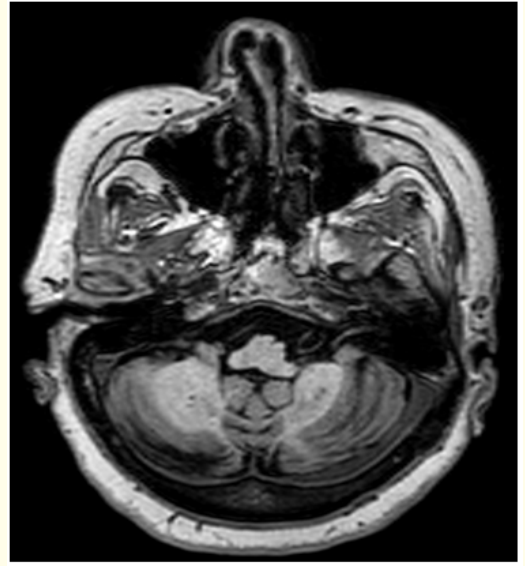

Figure 1: Axial noncontrast-enhanced Magnetization Prepared Rapid Acquisition Gradient Echo (MPRAGE) of the brain at the level of pons showing dilated basilar artery (white arrow) with an elongated aberrant course coursing toward the left cerebellopontine angle cistern and turning back to the midline causing compression upon the left anterolateral aspect of pons.
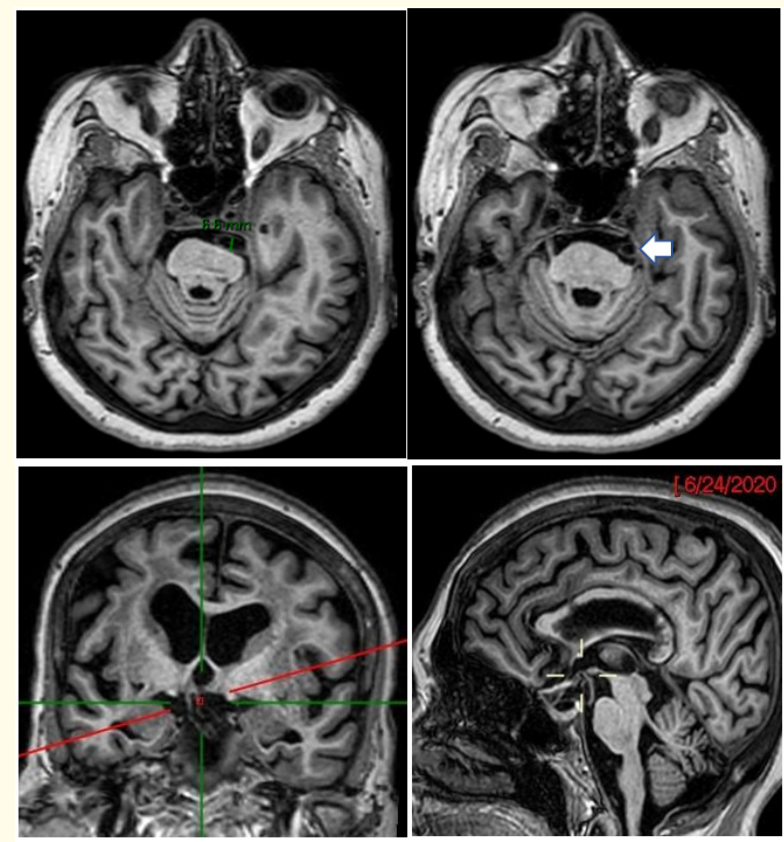

Figure 2: Axial noncontrast-enhanced Magnetization Prepared Rapid Acquisition Gradient Echo (MPRAGE) of the brain at the level of pons showing dilated basilar artery abutting the floor of the 3rd ventricle. 
Constructive Interference in Steady State (CISS) sequence (Figure 3) revealed that the ectatic vessel was associated with left-sided trigeminal nerve atrophy, with marked thinning of the preganglionic cisternal segment of the left trigeminal nerve. Mild displacement of the left facial nerve by the ectatic basilar artery was also appreciated.

The trigeminal nerve could not be visualized in left Meckel's cave and the left porus trigeminus (Figure 4). Rest of the visualized cranial nerves appeared normal. Being acquired in nature, the pathology was deduced to be nerve atrophy rather than nerve hypoplasia.

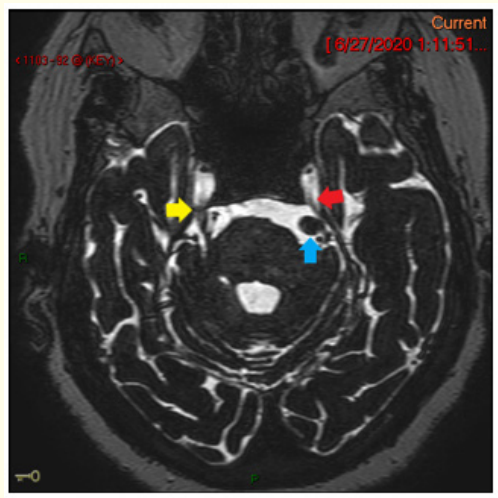

Figure 3: Axial Constructive Interference in Steady State (CISS) sequence) of the brain at the level of lower pons showing dilated basilar artery (blue arrow) with an elongated aberrant course coursing toward the left cerebellopontine angle cistern, a normal right trigeminal nerve (yellow arrow) and a hypoplastic left trigeminal nerve (red arrow).

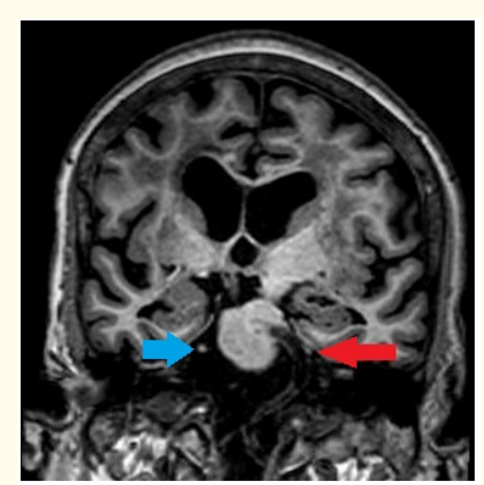

Figure 4: Coronal MPRAGE image at the level of Meckel's cave. Empty left Meckel's cave. Left trigeminal nerve (red arrow) appears atrophies and lateralized. Normal right trigeminal nerve

(blue arrow).

\section{Discussion}

Vertebrobasilar dolichoectasia has been associated with extrinsic cranial nerve compression, motor and sensory abnormalities, and obstructive hydrocephalus. The disorder has more male predilection and is seen usually in $6^{\text {th }}-7^{\text {th }}$ decade of life. The Smoker's criteria for dolichoectasia include: a diameter of more than 4.5 $\mathrm{mm}^{3}$, height of bifurcation at, or indenting and elevating, the floor of the third ventricle, and laterality of the ectatic vessel lateral to the lateral margin of the clivus or dorsum sellae or at the cerebellopontine cistern. It usually affects the basilar artery alone, and less commonly bilateral vertebral arteries, basilar artery and both vertebral arteries. Compression of cranial nerves can lead to hemifacial spasms, trigeminal neuralgias (when involving fifth cranial nerve), visual disturbances (when involving the optic tract) and nystagmus. The few cases of vertebrobasilar dolichoectasia complicated by trigeminal neuralgia that were described in the literature, where lacking obvious hypoplasia atrophy of the trigeminal nerve. The condition (vertebrobasilar dolichoectasia) was rather causing compression and thus irritation of the trigeminal nerve. In our case, the hemifacial anesthesia corresponded well with the imaging findings manifested by atrophy of the left trigeminal nerve, thought to be caused by chronic vascular compression. However, this clinical condition, in general, is rarely symptomatic [4].

Like this particular patient, this condition is associated with higher risk of cerebrovascular accident as VBD may promote brain ischemia, with occlusion of small perforating vessels, reduction of anterograde flow in the dilated artery, stress on and distortion and stretching of the branches of the basilar artery (Duret's Hemorrhages), and underlying atheromatous changes [5].

Management of vertebrobasilar dolichoectasia depends upon the clinical picture. It mainly involves eye care to lessen the effects of corneal anesthesia like eye dryness and keratoconjunctivitis, with treatment revolving around lubricating eye drops and ointments, eye patching, steroids, and in some cases, antibiotics. In cases associated with obstructive hydrocephalus, ventriculo-peritoneal shunting may be required to relieve the obstruction. Regular follow-up is recommended in most cases.

It is our opinion that practitioners should have a high degree of clinical suspicion when dealing with similar clinical presentations.

\section{Conclusion}

Vertebrobasilar dolichoectasia should be considered as a rare etiologic factor of trigeminal nerve atrophy not explained by other causes. 


\section{Bibliography}

1. Siddiqui A., et al. "Vertebrobasilar dolichoectasia: a rare cause of obstructive hydrocephalus: case report". British Institute of Radiology 81.964 (2008): e123.

2. B Ince., et al. "Dolichoectasia of the intracranial arteries in patients with first ischemic stroke: a population-based study". Neurology 50.6 (1998): 1694-1698.

3. S Love and HB Coakham. "Trigeminal neuralgia: pathology and pathogenesis". Brain 124.12 (2001): 2347-2360.

4. A De Azambuja Pereira-Filho., et al. "Brainstem compression syndrome caused by vertebrobasilar dolichoectasia". Arquivos de Neuro-Psiquiatria 66.2 (2008): 408-411.

5. S Passero and G Filosomi. "Posterior circulation infarcts in patients with vertebrobasilar dolichoectasia". Stroke 29.3 (1998): 653-659.

\section{Assets from publication with us}

- Prompt Acknowledgement after receiving the article

- Thorough Double blinded peer review

- Rapid Publication

- Issue of Publication Certificate

- High visibility of your Published work

Website: www.actascientific.com/

Submit Article: www.actascientific.com/submission.php

Email us: editor@actascientific.com

Contact us: +919182824667 